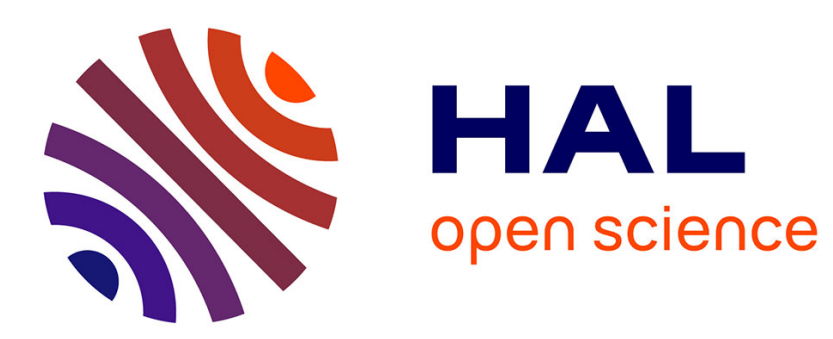

\title{
Exosome-mediated quality control: substrate recruitment and molecular activity
}

Alice Lebreton, Bertrand Séraphin

\section{To cite this version:}

Alice Lebreton, Bertrand Séraphin. Exosome-mediated quality control: substrate recruitment and molecular activity. BBA - Biochimica et Biophysica Acta, 2008, 1779 (9), pp.558-65. 10.1016/j.bbagrm.2008.02.003 . hal-01350691

\section{HAL Id: hal-01350691 \\ https://hal-ens.archives-ouvertes.fr/hal-01350691}

Submitted on 3 Aug 2016

HAL is a multi-disciplinary open access archive for the deposit and dissemination of scientific research documents, whether they are published or not. The documents may come from teaching and research institutions in France or abroad, or from public or private research centers.
L'archive ouverte pluridisciplinaire HAL, est destinée au dépôt et à la diffusion de documents scientifiques de niveau recherche, publiés ou non, émanant des établissements d'enseignement et de recherche français ou étrangers, des laboratoires publics ou privés. 


\title{
Exosome-mediated quality control: Substrate recruitment and molecular activity
}

\author{
Alice Lebreton, Bertrand Séraphin ${ }^{1}$ \\ Équipe Labellisée La Ligue, Centre de Génétique Moléculaire, CNRS UPR2167, \\ associée à l'Université P. et M. Curie, Avenue de la Terrasse, 91198 Gif sur Yvette, France.
}

Received 9 November 2007; accepted 5 February 2008

Available online 14 February 2008

doi:10.1016/j.bbagrm.2008.02.003

This is an accepted manuscript of an article published by Elsevier in Biochimica et Biophysica Acta - Gene regulatory mechanisms in September 2008, available online:

http://www.sciencedirect.com/science/article/pii/S187493990800045X.

\begin{abstract}
The eukaryotic exosome is a multisubunit complexes that is mainly responsible for 3' -5 ' exonucleolytic degradation of RNAs, both in the nucleus and the cytoplasm. In this review we summarize the recent experiments that have provided information on the organization, structure and activity of this large assembly. Interestingly, eukaryotic exosomes have been implicated in a large number of RNA degradation pathways including recently discovered RNA quality control mechanisms. A variety of cofactors have been shown to participate in substrate recruitment and/or assist exonucleolytic activities. Despite this avalanche of new results, further analyses will be required to improve our understanding of exosome regulation.
\end{abstract}

Keywords: Exosome; helicase; RNA quality control; RNA degradation; 3'-5' exoribonuclease; 3'-5' exoribonuclease; structure-function analysis.

\footnotetext{
${ }^{1}$ Corresponding author. Tel.: +33 1698238 84; fax: +331698238 77. E-mail address: seraphin@igbmc.fr.
} 


\section{Introduction}

RNA metabolism produce a variety of substrates targeted to degradation, including by-way products of RNA maturation (rRNA processing intermediates, debranched introns), non-functional mRNAs, intergenic polymerase II transcripts, abnormally folded non-coding RNAs or transcripts reaching the end of their lifespan. Several distinct mechanisms are involved in the degradation of these species, which mostly occurs exonucleolytically either from the 5' to the 3'-end, or from 3' to 5' [1]. Among the various nucleases implicated in these processes, the exosome assumes most of the 3'-5' decay. In the cytoplasm, its activity contributes to the "minor degradation pathway" for normal transcripts in yeast [2], and participates in the non-stop decay (NSD) [3] and nonsense mediated decay (NMD) [4] of non-functional mRNAs; conversely, in the nucleus, it is required for rRNA, snoRNA and snRNA processing [5], and constitutes the main component of the quality-control mechanisms which exerts on newborn or newly processed transcripts.

The exosome was first identified in yeast as a complex of multiple 3'-5' exonucleases [6]. The current model for the yeast exosome, as determined by recent structural and functional studies [7], consists of a conserved platform of 9 subunits, which shares similarity with the archaeal exosome and the bacterial PNPase, and of two catalytic subunits harbouring 3'-5' exonucleolytic activity, the RNase II-like Dis3, and the nuclear specific Rrp6. The composition of the human exosome appears to be quite similar to that of $S$. cerevisiae [8], though the homologue of Dis3 in higher eukaryotes does not seem to associate stably with the rest of the complex.

When measured in vitro, the intrinsic exonucleolytic activity of the exosome is quite low, but it can be stimulated by a number of nuclear or cytoplasmic specific cofactors, which were shown to interact with the core exosome subunits. These cofactors are thought to play a dual role in the degradation mechanism, on the one hand by selecting bona fide substrates for the exosome and on the other hand by stimulating the activity of the catalytic subunits.

We review here the latest advances made in the understanding of the exosome structure and general mechanism of action. Given the large diversity of the transcripts that undergo 3'-5' degradation, one might wonder how they are selected within the population of cellular RNAs, and specifically orientated towards the exosome. We thus focus on the recruitment mechanisms that target substrates recognized for various features to exosome-mediated degradation.

\section{Exosome structure}

Eukaryotic exosome subunits were first identified genetically in the yeast Saccharomyces cerevisiae in independent screens for mutants defective in rRNA maturation [9], accumulation of poly(A) RNA in the nucleus [10] or expression of plasmid encoded toxins [11]. These original observations provided entry points for further genetic and biochemical analyses, which revealed the existence of a large complex that was named the exosome [6]. Analysis of the sequence of several of its subunits revealed similarities to subunits of bacterial PNPase/RNase PH or RNase II. Further analysis demonstrated that the exosome is highly conserved in eukaryotic cells. Interestingly, the exosome was found to be present in both the nuclear and cytoplasmic compartments even though its compositions differed slightly.

These original pieces of work have led to our current view of exosome [12,13]. The yeast exosome is composed of a conserved core of 10 subunits (Rrp4, Rrp40, Cs14, Ski6/Rrp41, Rrp42, Rrp43, Rrp45, Rrp46, Mtr3, Dis3/Rrp44) that is present both in the nucleus and the cytoplasm. In the cytoplasm, the core associates to the Ski7 protein that has similarity to translation elongation factor 1 to form the full exosome structure specific of this compartment. In contrast, the core binds Rrp6, a protein with similarity to bacterial RNase D and the Lrp1/Rrp47 factor, to form the full nuclear exosome. It is important to note, however, that these descriptions are not definitive. Indeed, it is currently unclear whether the two different forms of the exosome are totally stable entities or whether they represent dynamic assemblies where exchange between subunits may occur with time in vivo. Thus, the same core exosome unit could possibly shuttle between the nucleus and the cytoplasm and associate with specific subunits according to the compartment in which it resides. Reciprocally, there is no clear evidence that compartment specific subunits are only present and active as part of full exosomes. Even further, the yeast core exosome could itself represent a dynamic assembly. This is less likely though given that, with the exception of Dis3 and/or Csl4, it appears to be extremely stable in vitro [14].

Given the good conservation of the exosome subunits, it is widely accepted that the yeast exosome provides a good model for eukaryotic species. Purification and analysis of the human exosome suggest that some differences may however exist. For instance, affinity selection of the human exosome suggests that the Dis3/Rrp44 subunits may at most only be weakly associated to the 9 other core subunits [15]. Even though this is not yet definitive, localisation data suggest that PM/Scl-100, the human homologue of Rrp6, could be present both in the nucleus and cytoplasm of human cells [16]. Finally, a nuclear specific subunit of the human exosome has been characterized, MPP6, for which no homologue has been identified in yeast so far [17].

In addition to uncertainties concerning the exact composition and dynamics of the full cytoplasmic and nuclear exosomes, the stoichiometry and organisation of the core exosome was also unclear for a long time. Indeed, 
biochemical fractionation and interaction studies could not discriminate between the presence of a mixture of complexes containing different subunits in varying stoichiometry or the presence of a single well-defined assembly. Two-hybrid analyses and purification of subunits co-expressed in E. coli suggested that the various subunits associated in a limited number of possible manners [18-20]. Sequence comparisons had revealed similarities between 9 eukaryotic core exosome subunits and various domains of the bacterial PNPase/degradosome, RNase PH and subunits of archaeal exosome. Interestingly, these complexes have all been shown to be endowed with 3'-5' phosphorolytic nuclease activity. Based on these sequence similarities, it was suggested that the eukaryotic exosome adopted a similar shape with 6 subunits harbouring a RNase PH fold (namely Rrp41/Ski6, Rrp42, Rrp43, Rrp45, Rrp46 and/or Mtr3) forming a ring structure and 3 subunits containing RNA binding domains (namely Rrp4, Rrp40 and/or Cs14) lying on top [21, 22]. This proposal was supported by electron microscopy analysis that confirmed the general organisation as a ring structure but did not allow the definition of the exact subunit arrangement [21]. These ambiguities were solved by a native mass spectrometry of purified core exosome [14]. This study demonstrated that the yeast core exosome had a defined stoichiometry with each of the 10 subunits being present in a single copy. Moreover, analysis of subcomplexes generated by the dissociation of core exosome complexes allowed the mapping of the exact subunit interaction pattern, which differed significantly from proposals based on structural modelling or some large-scale interaction analyses [21, 23]. The structural model of the yeast core exosome was recently confirmed by the impressive reconstitution of the ring part of the human core exosome using 9 recombinant subunits and the resolution of its structure by X-ray diffraction [8]. This study validated the subunit organisation scheme and provided long-awaited details about sequences insuring specific subunit interactions. The location of Dis 3 relative to the ring subunits remains only poorly defined. Data obtained by native mass spectrometry of the yeast core exosome gave some preliminary idea of the partners of this large subunit but its exact arrangement remained imprecise [14]. Recent electron microscopy analyses, combined with the structure of bacterial RNase II (which is similar to Dis3) $[24,25]$ have provided a more complete model of the yeast core exosome [26]. It is expected that further details will be obtained through the structural analysis of Dis3 alone and/or in combination with other core subunits.

Analysis of the interaction pattern of subunits specifically present in the nuclear or cytoplasmic full exosome, or regulatory factors, is clearly lagging behind. Figure 1 summarizes information currently obtained by a variety of approaches including two-hybrid studies, affinity purifications and biochemical analyses. Again, in the near future, structural analysis of these factors as independent subunits or in complexes will probably provide us with a better understanding of the interactions occurring in this dynamic network. Such data have, for example, already been obtained for subdomains of the yeast Rrp6 protein confirming the presence of an RNase D fold in this protein endowed with 3'-5' hydrolytic activity [27].

\section{Catalysis of RNA degradation by the eukaryotic exosome}

Given the similarity of the ring part of the human and yeast exosomes to bacterial PNPase/degradosome, RNase PH or archaeal exosome, the eukaryotic exosome was long thought to be endowed with 3'-5' phosphorolytic activity. This idea was supported by early activity analysis with recombinant subunits for which phosphorolytic activities, albeit weak, were reported [6]. However, a detailed analysis of the activity of highly purified yeast core exosome failed to support this model [7, 8]. Instead, Dis3 was observed to be responsible for all 3'-5' exonucleolytic activity of the yeast core exosome, both in vitro and in vivo. This hydrolytic exonuclease activity was abolished by a single point mutation in the RNase II-type catalytic site of Dis3. Consistently, early assays performed with material immunoprecipitated from yeast had also revealed the exclusive presence of a hydrolytic activity [9]. The data obtained with the yeast core exosome suggested that the 9 -member ring structure was thus inactive. Analysis of the homologous human structure produced recombinantly has demonstrated that it is also inactive $[8,28]$. It is however possible that this feature does not apply to all eukaryotic exosomes as analysis of a highly purified recombinant plant exosome subunit suggests that it can catalyze RNA degradation in a phosphorolytic reaction [29].

If Dis3 appears to be the main catalytic subunit of the core exosome, its action is strengthened in the nucleus by Rrp6 that is also endowed with $3^{\prime}-5$ ' hydrolytic exonuclease activity [30, 31]. Indeed, structural analysis of a fragment of the yeast Rrp6 protein has provided some insights into its interaction with reaction products [27]. It is currently unclear, however, neither how the activities of Dis3 and Rrp6 in RNA degradation are coordinated nor whether they cooperate to degrade given substrates. Alternatively, one could think that each activity is specialized for the degradation of specific targets. Moreover, the mode of interaction of RNA substrates with the exosome remains uncertain. On the one hand, evidence for direct interaction of substrate RNA with Dis 3 has been provided [32]. On the other hand, the role of the inactive 9-mer ring subunits, which have been proposed to channel substrate RNAs to the active site of archaeal exosomes [33], remains mysterious as, despite their lack of detectable catalytic activity, each one of these 9 subunits has been shown to be essential for viability in yeast. Further work should certainly reveal the role of the various subunits and catalytic sites of eukaryotic exosome in substrate degradation. 


\section{Exosome-mediated degradation pathways}

Considering the variety of transcripts that undergo exosome-mediated, 3'-5' decay, several distinct pathways are likely to detect and target substrates towards the exosomal scaffold. Indeed, a number of experimental evidence indicate that, depending on its nature and sub-cellular localization, each substrate is recognized by specific exosome cofactors, which in turn determine its fate. Here, we aim to give an overview of various substrate recognition strategies and the cofactors they involve, then to define some common features between pathways; more complete descriptions of each mechanism can be found in recent reviews of the topic [1,34-36] or in this issue. Figure 1 recapitulates the involvement of the known exosome cofactors in distinct nuclear and cytoplasmic degradation pathways.

\subsection{Nuclear quality control mechanisms}

In $S$. cerevisiae, a large part of the nuclear quality control on nascent transcripts relies on the activity of a complex called TRAMP (for Trf4/5-Air1/2-Mtr4 Polyadenylation complex) [37, 38]. This control affects a wide range of RNA species, among which hypomodified tRNAs [39], precursors or abnormal maturation intermediates of snoRNAs, snRNAs and rRNAs [37, 40,41], as well as the so-called CUTs (Cryptic Unstable Transcripts), a category of newly described, very short half-lived intergenic RNA polymerase II transcripts [38, 42]. Such abnormal RNAs are virtually absent from wild type cells, and accumulate in TRAMP and exosome mutants.

TRAMP complexes constitute of a non-canonical poly(A) polymerase (Trf4 or Trf5), associated with a zinc knuckle protein (Air1 or Air2), and a RNA helicase of the DExD/H family (Mtr4). Air1/2 proteins are thought to be involved in the binding of the RNA substrates, thus permitting their polyadenylation by Trf4/5. TRAMP activity is followed by the complete degradation of the transcripts by the exosome, a process which evocates the recruitment of the degradosome to polyadenylated substrates in prokaryotes $[12,43]$.

In exosome mutant strains, polyadenylated forms of abnormal RNAs accumulate $[37,38]$. Moreover, the stimulation of the activity of the exosome by TRAMP is reduced in Trf4 catalytic site mutants [38]. Nevertheless, the mere polyadenylation of the substrate is not sufficient for efficient degradation. Indeed, purified exosome preparations were shown to deadenylate in vitro adenylated tRNAs, but a complete, processive degradation required the addition of purified TRAMP complex, and could be obtained even in the absence of ATP [37]. This experiment suggests that TRAMP cooperates with the exosome to stimulate its exonucleolytic activities, through direct interactions and independently of polyadenylation. These data do not exclude, however, that in vivo, several rounds of TRAMPmediated polyadenylation followed by exosome trimming might be required for the complete processing of structured substrates.

Aside from this pathway, a fraction of the nuclear exosome substrates fall into TRAMP-independent decay mechanisms, as the 7S rRNA intermediate, the processing of which leads to the formation of 5.8S rRNA [6], and the 5 '-ETS (External Transcribed Spacer) resulting from the cleavage of the $35 \mathrm{~S}$ pre-rRNA precursor at sites $\mathrm{A}_{0}$, which is eliminated by the exosome. Mtr4, but not the other TRAMP components, appears to be required for the recruitment of the exosome to these transcripts [44].

Homologues of TRAMP components can be found in other eukaryotes, where they are likely to play the same function. A complex similar to TRAMP has been identified in $S$. pombe, where it participates in gene silencing through nuclear polyadenylation activities $[45,46]$. The human genome also displays close homologues of Trf4/5 [47] and Air1/2, as well as one homologue of Mtr4, hMtr4, which is required for rRNA processing [48]. However, whether these components associate to form human TRAMP complexes is still an open question.

\subsection{Cytoplasmic quality control mechanisms}

Even if the major cytoplasmic RNA degradation pathway occurs from 5' to 3', in a process involving Xrn1, the cytoplasmic exosome has been shown to participate in a variety of 3'-5' degradation activities such as mRNA turnover [2], NSD [3] and NMD [4]. In all these pathways, the yeast exosome is activated by Ski7 [49, 50], a small GTPase belonging to the family of translation elongation factors EF1 $\alpha$, and the Ski complex [2]. The latter has been proposed to be a hetero-tetramer constituted by the Ski2 DExH-box putative helicase, Ski3 and two copies of Ski8 $[51,52]$. The interaction of Ski7, through its N-terminal region, with both the exosome and the Ski complex, was shown to be essential for the 3'-5' decay of mRNAs [50]. So far, little is known about the molecular mechanisms that allow the activation of the exosome by Ski7 and the Ski complex.

The detection of the substrates is slightly better understood. For instance, in the yeast NMD mechanism, loading of the Upf1/2/3 surveillance complex to a mRNA with a premature termination codon, followed by binding of Ski7 to Upf1 via its N-terminal domain, allows the recruitment of the 3'-5' decay machinery [4, 53]. Ski7 is also thought to allow the recognition of mRNAs devoid of a stop codon when ribosomes reach the 3'-endby occupying the empty A site. This interaction is permitted by the structural similarity of the Ski7 GTPase with both eEF1A and eFR3, which respectively brings aminoacylated tRNAs to sense codons during translation elongation, or allows translation termination when the ribosome encounters a stop codon. 
In mammalian cells, the exosome, together with the 5' -3 ' decay pathway, is also involved in the regulation of the levels of certain transcripts by a sequence-based recognition. The ARE-mediated decay (AMD) sends AU-Rich Elements (ARE)-containing transcripts towards degradation, through their recognition by ARE-binding proteins (ARE-BPs), such as BRF-1, TTP and KSRP [54, 55], which interact directly with the cellular decapping, deadenylation and decay machineries [56]. As for general mRNA turnover, exosome-mediated AMD seems to affect a minor fraction of transcripts, the major part undergoing the major 5'-3' decay pathway [57].

\subsection{The role for RNA helicases in 3'-5' decay}

A common feature between all 3'-5' decay pathways seems to be the requirement for cofactors belonging to the DExD/H-box family of RNA helicase. Strikingly, this is not typical of eukaryotic mechanisms, since the DEAD-box helicase RhlB, though not essential for viability, is an important component of E. coli degradosome [12]. In yeast, the essential nuclear DExH-box protein Mtr4, and its cytoplasmic counterpart Ski2, are respective cofactors of the nuclear and cytoplasmic forms of the exosome.

Strong evidence indicates that Mtr4 is required for both TRAMP-dependent and independent activities of the nuclear exosome [41,44], with the exception of the final trimming of structured substrates such as $5.8 \mathrm{~S}+30$ and certain pre-snoRNAs that might require the activity of Rrp6 alone, through the involvement of its HDRC domain (helicase RNase D C-terminal domain) [34,58].

Regardless of its central role in nuclear exosome function, the precise mechanism of action of Mtr4 is still unclear. Its RNA-dependent ATPase activity, as well as its ability to unwind RNA duplexes, was recently reported [59]. It is thus tempting to speculate that this RNA helicase would unwind structures in RNA substrates in order to allow exosome progression. Like other members of the family [60, 61], Mtr4 might also act as an RNPase, assisting the exosome by removing proteins from RNA molecules without duplex unwinding. Alternately, Mtr4 could also mediate the recruitment of the exosome to substrates through direct protein-RNA and protein-protein interactions, independently of any helicase activity.

Functional hints regarding the mechanism of action of Mtr4 could be provided by recent data obtained for Suv3, which is the RhlB homologue in the degradosome counterpart of eukaryotic mitochondria [62]. The mitochondrial helicase and RNase components of the degradosome prove to tightly cooperate, leading the authors to propose a model where the helicase moiety of the complex would act as a molecular motor feeding the nuclease with RNA substrates.

\section{Substrate recognition mechanisms: sequence versus structure}

The mechanisms resulting in the targeting of substrates towards the exosome are diverse, and highly depend on the nature of the transcript. Here we try to point out a few prototypic mechanisms resulting in the processing of definite classes of transcripts.

\subsection{Sequence-based mechanisms of substrate recruitment}

Certain classes of transcripts are recognized through sequence-based mechanisms, as was described for AMD in the previous paragraph. Apart from RNAs containing AU-rich elements in their 3'-UTRs, transcripts containing Nrd1 or Nab3 binding sites would also undergo such mechanisms.

Nrd1 and Nab3 are yeast RNA-binding proteins involved, together with the helicase Sen1, in the 3'-endformation of non-poly(A)-polymerase II transcripts such as snRNAs and snoRNAs [63, 64]. Binding of Nrd1 and Nab3 to their specific sites (GUA[A/G] and UCUU, respectively) mediates transcript termination through a polyadenylationindependent mechanism; the formation of heterodimers allows their cooperative recruitment to transcripts containing multiple binding sites [65]. Similar 3'-endformation mechanisms account for the termination of CUTs $[64,66,67]$. Nab3-Nrd1 dependent termination was shown to be required, though not sufficient, for the elimination of CUTs mediated by TRAMP and the exosome [65,67]. Since Nrd1 and Nab3 can interact with the nuclear form of the exosome [68], these proteins are proposed to link the transcription and nuclear RNA quality control, through a direct recruitment of substrates as their 3'-endis being formed.

It is still unclear whether the Nab3/Nrd1 termination of transcripts is an active process, or whether this constitutes a "by default" mechanism, affecting transcripts devoid of regular cleavage and polyadenylation sites. Both hypotheses are likely to occur on distinct classes of RNAs, the former being required for the 3'-endformation of snRNAs and snoRNAs, the latter permitting the degradation of aberrantly transcribed species such as CUTs, when Nab3/Nrd1 sites are encountered more or less randomly within the sequence. The question is still open about how the choice between partial trimming or triggering of a complete degradation of transcripts operates. The density of Nrd1 and $\mathrm{Nab} 3$ sites at the 3'-endof transcripts might be the keystone of the problem, by promoting a more or less intense nucleation of Nab3-Nrd1 heterodimers on 3'-ends [65]. One of the proposed mechanisms suggests that the progression of the nuclear exosome would be blocked by the structures of correctly folded RNPs, and/or by the $\mathrm{Nab3/Nrd1} \mathrm{complexes,} \mathrm{hence} \mathrm{resulting} \mathrm{in} \mathrm{the} \mathrm{formation} \mathrm{of} \mathrm{proper} \mathrm{3'-ends} \mathrm{in} \mathrm{snRNA} \mathrm{and} \mathrm{snoRNAs;} \mathrm{otherwise,} \mathrm{the}$ 
degradation would be complete $[67,68]$. The effective role of the TRAMP complex in this process also remains poorly understood.

Sequence-specific recruitment of the exosome is also involved in rRNA processing events. In vitro, the human Mphase phosphoprotein 6 (MPP6) was reported to bind preferentially pyrimidin-rich RNAs, and displays affinity for the internal transcribed spacer 2 (ITS2) in rRNA precursors [17]. In vivo, the knockdown of its expression results in defects in 5.8S rRNA 3'-endformation. MPP6 is proposed to mediate the recruitment of the nuclear exosome to a CCCCUUCCCCCUCCCCCC sequence within the ITS2 [17], together with KIAA052, C1D and PM/Scl-100 (the human homologues of Mtr4, Lrp1 and Rrp6, respectively) [48].

\subsection{Structured-based mechanisms of substrate recruitment}

Independently of their sequence, some transcripts appear to be rather orientated towards the exosome according to their structural properties. Two distinct processes can be defined: on the one hand, abnormally folded RNAs have to be detected and eliminated by quality control mechanisms; on the other hand, certain classes of transcripts harbouring typical structural motifs can also undergo exosome-mediated degradation.

Abnormally folded RNA species appear to be recognized in yeast by the TRAMP complex, which enables their complete degradation by the exosome, instead of a partial trimming. The precise mechanisms involved in the detection of these species are still hypothetical. One possible explanation relies on a proposed model for the degradation of prokaryotic RNA [69]. Abnormally folded RNA species would expose protruding 3'-ends, accessible for polyadenylation, and would then be processed from $3^{\prime}$ to $5^{\prime}$ by the polynucleotide phosphorylase (PNPase). On the contrary, fully functional RNAs would be structured so that these ends would be buried into the structure of RNPs, and as a consequence protected from degradation. Based on the thermodynamic instability of a number of TRAMP-exosome substrates $[39,70]$, a similar model has been proposed for the TRAMP-mediated quality control [35].

Another possible mechanism is that thermodynamically stable secondary structures, or association to proteins within RNPs, would block the progression of the exosome on certain transcripts, and thus orientate their fate, by operating the choice between a partial trimming and a complete degradation. Such a mechanism has been anticipated for the maturation of snRNAs and snoRNAs as reported above [68], as well as for the control of the 5.8S rRNA 3'endformation from its $7 \mathrm{~S}$ precursor [5].

The specific addressing of structured substrates to the exosome is likely to involve specific cofactors. The yeast protein Lrp1/Rrp47, or its homologue in higher eukaryotes C1D, is probably one of them. This factor binds RNAs with a preferential specificity for structured species [48, 71]. Rrp47 was also reported to form a hexameric complex, which interacts with the PMC2NT of Rrp6 and stimulates its activity [71, 72]. It is therefore supposed to promote the degradation of Rrp6 substrates containing structured motifs such as tRNAs and snRNAs, as well as the potential RNA duplexes created by the hybridization of antisense CUTs with target mRNAs [71].

\section{Conclusion}

Given its barrel-like structure, the exosome has sometimes been compared to the proteasome, as a macromolecular cage devoted to RNA degradation [13]. Nevertheless, though the global architecture of the ring is conserved between the prokaryotic and eukaryotic phyla, the location of the nucleolytic centers prove to have diverged across evolution; if these activities are buried inside the central channel in both the archaeal exosome and the bacterial degradosome, they reside on more labile subunits - Dis3 and Rrp6 - in several of their eukaryotic counterparts [28]. Henceforth, the stable macromolecular structure adopted by the 9 ring subunits appears more like a central scaffold, which may coordinate the activities of the nucleases and potential regulation mechanisms. An appealing hypothesis is that the ring subunits may control the accessibility of substrates to the catalytic sites due to steric considerations, hence limiting the nucleolytic capacities of the RNAses compared to free subunits [26]. The macromolecular structure could also contribute to the reaction by gathering in close vicinity the substrates, the exonucleases and various cofactors.

If the molecular function of most cofactors is still unclear, they turn out to play a major part in the process, either by selecting the substrates, or by assisting the exonucleases. A large part of the future works aiming at understanding the exosome mode of action will probably concentrate on solving the exact roles of these cofactors. Apart from the main ones cited above, which display a physical association with exosome components, other partners may influence exosome activity; among them, the Ran GTPase Gsp1 is likely to play a crucial role in the quality control of precursors of ribosomal subunits. Indeed, mutants of this protein display 5.8S maturation defects very similar to that of Dis3 mutants, and can be compensated by overexpression of either Dis3 or Mtr4 [73]. Considering the role of Gsp1 in the export of pre-ribosomal subunits from the nucleus to the cytoplasm, this protein might bridge the quality control of the ribosomal RNAs with the regulation of export.

In addition to quality control mechanisms described here, the exosome and its cofactors were shown to participate in other cellular processes, such as regulations of specific mRNA levels. A few such regulatory pathways have been described to date, including the autoregulation of NRD1 mRNA levels by degradation induced by premature 
transcription termination [74], the control of transcription of the IMD2 locus by short, polyadenylated, promoterassociated transcripts that are substrates for the exosome [75], or the regulation of histone mRNA amounts by TRAMP-exosome mediated decay [76]. Recent evidence also suggest that the exosome may participate in gene silencing mechanisms; for instance, in Schizosaccharomyces pombe, polyadenylation of transcripts produced within centromeric repeats by $\mathrm{Cid} 14$, which show sequence similarity to $\mathrm{Trf} 4 / 5$, results in gene silencing by recruitment of the exosome or the RNAi machinery [45]. In the unicellular green alga Chlamydomonas reinhardtii, MUT68 ${ }_{\mathrm{p}}$, a poly(A)-polymerase of the same family, polyadenylates RNA intermediates resulting from siRNA-directed cleavage, thus stimulating exosome-mediated degradation [77]. Altogether, a number of functional data converge towards a view in which the exosome would be the final end of a large variety of pathways involved both in quantitative and qualitative RNA quality controls.

\section{Acknowledgements}

We thank Christopher D. Lima for sending his latest manuscript prior to publication. This work was supported by the CNRS, La Ligue contre le Cancer (Equipe labellisée 2005), l'Agence Nationale pour la Recherche (project ANR-05- BLAN-0062-01), the European Science Fundation Eurocores (programme RNA Quality, project EUxosome) and the EU Sixt Framework Program (LSHG-CT-2005-512028).

\section{Reference list}

[1] O. Isken, L.E. Maquat, Quality control of eukaryotic mRNA: safeguarding cells from abnormal mRNA function, Genes Dev 21 (2007) 1833-3856.

[2] J.S. Anderson, R.P. Parker, The 3' to 5' degradation of yeast mRNAs is a general mechanism for mRNA turnover that requires the SKI2 DEVH box protein and 3' to 5' exonucleases of the exosome complex, Embo J 17 (1998) 1497-1506.

[3] A. van Hoof, P.A. Frischmeyer, H.C. Dietz, R. Parker, Exosome-mediated recognition and degradation of mRNAs lacking a termination codon, Science 295 (2002) 2262-2264.

[4] P. Mitchell, D. Tollervey, An NMD pathway in yeast involving accelerated deadenylation and exosomemediated 3'-->5' degradation, Mol Cell 11 (2003) 1405-1413.

[5] C. Allmang, J. Kufel, G. Chanfreau, P. Mitchell, E. Petfalski, D. Tollervey, Functions of the exosome in rRNA, snoRNA and snRNA synthesis, Embo J 18 (1999) 5399-5410.

[6] P. Mitchell, E. Petfalski, A. Shevchenko, M. Mann, D. Tollervey, The exosome: a conserved eukaryotic RNA processing complex containing multiple 3'-->5' exoribonucleases, Cell 91 (1997) 457-466.

[7] A. Dziembowski, E. Lorentzen, E. Conti, B. Seraphin, A single subunit, Dis3, is essentially responsible for yeast exosome core activity, Nat Struct Mol Biol 14 (2007) 15-22.

[8] Q. Liu, J.C. Greimann, C.D. Lima, Reconstitution, activities, and structure of the eukaryotic RNA exosome, Cell 127 (2006) 1223-1237.

[9] P. Mitchell, E. Petfalski, D. Tollervey, The 3' end of yeast 5.8S rRNA is generated by an exonuclease processing mechanism, Genes \& development 10 (1996) 502-513.

[10] S. Liang, M. Hitomi, Y.H. Hu, Y. Liu, A.M. Tartakoff, A DEAD-box-family protein is required for nucleocytoplasmic transport of yeast mRNA, Mol Cell Biol 16 (1996) 5139-5146.

[11] S.P. Ridley, S.S. Sommer, R.B. Wickner, Superkiller mutations in Saccharomyces cerevisiae suppress exclusion of M2 double-stranded RNA by L-A-HN and confer cold sensitivity in the presence of M and LA-HN, Mol Cell Biol 4 (1984) 761-770.

[12] A.J. Carpousis, The RNA Degradosome of Escherichia coli: An mRNA-Degrading Machine Assembled on RNase E, Annu Rev Microbiol 61 (2007) 71-87.

[13] S. Lin-Chao, N.T. Chiou, G. Schuster, The PNPase, exosome and RNA helicases as the building components of evolutionarily-conserved RNA degradation machines, J Biomed Sci (2007).

[14] H. Hernandez, A. Dziembowski, T. Taverner, B. Seraphin, C.V. Robinson, Subunit architecture of multimeric complexes isolated directly from cells, EMBO Rep 7 (2006) 605-610.

[15] C.Y. Chen, R. Gherzi, S.E. Ong, E.L. Chan, R. Raijmakers, G.J. Pruijn, G. Stoecklin, C. Moroni, M. Mann, M. Karin, AU binding proteins recruit the exosome to degrade ARE-containing mRNAs, Cell 107 (2001) 451-464.

[16] E.L. van Dijk, G. Schilders, G.J. Pruijn, Human cell growth requires a functional cytoplasmic exosome, which is involved in various mRNA decay pathways, Rna 13 (2007) 1027-1035.

[17] G. Schilders, R. Raijmakers, J.M. Raats, G.J. Pruijn, MPP6 is an exosome-associated RNA-binding protein involved in 5.8S rRNA maturation, Nucleic Acids Res 33 (2005) 6795-6804.

[18] B. Lehner, C.M. Sanderson, A protein interaction framework for human mRNA degradation, Genome Res 14 (2004) 1315-1323.

[19] E. Lorentzen, P. Walter, S. Fribourg, E. Evguenieva-Hackenberg, G. Klug, E. Conti, The archaeal exosome core is a hexameric ring structure with three catalytic subunits, Nat Struct Mol Biol 12 (2005) 575-581. 
[20] A.M. Estevez, B. Lehner, C.M. Sanderson, T. Ruppert, C. Clayton, The roles of intersubunit interactions in exosome stability, J Biol Chem 278 (2003) 34943-34951.

[21] P. Aloy, F.D. Ciccarelli, C. Leutwein, A.C. Gavin, G. Superti-Furga, P. Bork, B. Bottcher, R.B. Russell, A complex prediction: three-dimensional model of the yeast exosome, EMBO reports 3 (2002) 628-635.

[22] M.F. Symmons, M.G. Williams, B.F. Luisi, G.H. Jones, A.J. Carpousis, Running rings around RNA: a superfamily of phosphate-dependent RNases, Trends in biochemical sciences 27 (2002) 11-18.

[23] A.C. Gavin, P. Aloy, P. Grandi, R. Krause, M. Boesche, M. Marzioch, C. Rau, L.J. Jensen, S. Bastuck, B. Dumpelfeld, A. Edelmann, M.A. Heurtier, V. Hoffman, C. Hoefert, K. Klein, M. Hudak, A.M. Michon, M. Schelder, M. Schirle, M. Remor, T. Rudi, S. Hooper, A. Bauer, T. Bouwmeester, G. Casari, G. Drewes, G. Neubauer, J.M. Rick, B. Kuster, P. Bork, R.B. Russell, G. Superti-Furga, Proteome survey reveals modularity of the yeast cell machinery, Nature 440 (2006) 631-636.

[24] C. Frazao, C.E. McVey, M. Amblar, A. Barbas, C. Vonrhein, C.M. Arraiano, M.A. Carrondo, Unravelling the dynamics of RNA degradation by ribonuclease II and its RNA-bound complex, Nature 443 (2006) 110114.

[25] Y. Zuo, H.A. Vincent, J. Zhang, Y. Wang, M.P. Deutscher, A. Malhotra, Structural basis for processivity and single-strand specificity of RNase II, Molecular cell 24 (2006) 149-156.

[26] H.W. Wang, J. Wang, F. Ding, K. Callahan, M.A. Bratkowski, J.S. Butler, E. Nogales, A. Ke, Architecture of the yeast Rrp44 exosome complex suggests routes of RNA recruitment for 3' end processing, Proceedings of the National Academy of Sciences of the United States of America 104 (2007) 1684416849.

[27] S.F. Midtgaard, J. Assenholt, A.T. Jonstrup, L.B. Van, T.H. Jensen, D.E. Brodersen, Structure of the nuclear exosome component Rrp6p reveals an interplay between the active site and the HRDC domain, Proc Natl Acad Sci U S A 103 (2006) 11898-11903.

[28] Q. Liu, J.C. Greimann, C.D. Lima, Reconstitution, Activities, and Structure of the Eukaryotic RNA Exosome, Cell 131 (2007) 188-189.

[29] J.A. Chekanova, R.J. Shaw, M.A. Wills, D.A. Belostotsky, Poly(A) tail-dependent exonuclease AtRrp41p from Arabidopsis thaliana rescues 5.8 S rRNA processing and mRNA decay defects of the yeast ski6 mutant and is found in an exosome-sized complex in plant and yeast cells, The Journal of biological chemistry 275 (2000) 33158-33166.

[30] M.W. Briggs, K.T. Burkard, J.S. Butler, Rrp6p, the yeast homologue of the human PM-Scl 100-kDa autoantigen, is essential for efficient 5.8 S rRNA 3' end formation, The Journal of biological chemistry 273 (1998) 13255-13263.

[31] K.T. Burkard, J.S. Butler, A nuclear 3'-5' exonuclease involved in mRNA degradation interacts with Poly(A) polymerase and the hnRNA protein Npl3p, Molecular and cellular biology 20 (2000) 604-616.

[32] C. Schneider, J.T. Anderson, D. Tollervey, The exosome subunit Rrp44 plays a direct role in RNA substrate recognition, Mol Cell 27 (2007) 324-331.

[33] E. Lorentzen, A. Dziembowski, D. Lindner, B. Seraphin, E. Conti, RNA channelling by the archaeal exosome, EMBO Rep 8 (2007) 470-476.

[34] J. Houseley, J. LaCava, D. Tollervey, RNA-quality control by the exosome, Nat Rev Mol Cell Biol 7 (2006) 529-539.

[35] K.M. Reinisch, S.L. Wolin, Emerging themes in non-coding RNA quality control, Curr Opin Struct Biol 17 (2007) 209-214.

[36] S. Vanacova, R. Stefl, The exosome and RNA quality control in the nucleus, EMBO Rep 8 (2007) 651657.

[37] J. LaCava, J. Houseley, C. Saveanu, E. Petfalski, E. Thompson, A. Jacquier, D. Tollervey, RNA degradation by the exosome is promoted by a nuclear polyadenylation complex, Cell 121 (2005) 713-724.

[38] F. Wyers, M. Rougemaille, G. Badis, J.C. Rousselle, M.E. Dufour, J. Boulay, B. Regnault, F. Devaux, A. Namane, B. Seraphin, D. Libri, A. Jacquier, Cryptic pol II transcripts are degraded by a nuclear quality control pathway involving a new poly(A) polymerase, Cell 121 (2005) 725-737.

[39] S. Vanacova, J. Wolf, G. Martin, D. Blank, S. Dettwiler, A. Friedlein, H. Langen, G. Keith, W. Keller, A new yeast poly(A) polymerase complex involved in RNA quality control, PLoS Biol 3 (2005) e189.

[40] D.E. Egecioglu, A.K. Henras, G.F. Chanfreau, Contributions of Trf4p- and Trf5p-dependent polyadenylation to the processing and degradative functions of the yeast nuclear exosome, Rna 12 (2006) 26-32.

[41] J. Houseley, D. Tollervey, Yeast Trf5p is a nuclear poly(A) polymerase, EMBO Rep 7 (2006) 205-211.

[42] C.A. Davis, M. Ares, Jr., Accumulation of unstable promoter-associated transcripts upon loss of the nuclear exosome subunit Rrp6p in Saccharomyces cerevisiae, Proc Natl Acad Sci U S A 103 (2006) 3262-3267.

[43] M. Dreyfus, P. Regnier, The poly(A) tail of mRNAs: bodyguard in eukaryotes, scavenger in bacteria, Cell 111 (2002) 611-613. 
[44] J. de la Cruz, D. Kressler, D. Tollervey, P. Linder, Dob1p (Mtr4p) is a putative ATP-dependent RNA helicase required for the 3' end formation of 5.8S rRNA in Saccharomyces cerevisiae, Embo J 17 (1998) 1128-1140.

[45] M. Bühler, W. Haas, S.P. Gygi, D. Moazed, RNAi-dependent and -independent RNA turnover mechanisms contribute to heterochromatic gene silencing, Cell 129 (2007) 707-721.

[46] S.W. Wang, A.L. Stevenson, S.E. Kearsey, S. Watt, J. Bahler, Global role for polyadenylation-assisted nuclear RNA degradation in posttranscriptional gene silencing, Mol Cell Biol 28 (2008) 656-665.

[47] C. Walowsky, D.J. Fitzhugh, I.B. Castano, J.Y. Ju, N.A. Levin, M.F. Christman, The topoisomerase-related function gene TRF4 affects cellular sensitivity to the antitumor agent camptothecin, J Biol Chem 274 (1999) 7302-7308.

[48] G. Schilders, E. van Dijk, G.J. Pruijn, C1D and hMtr4p associate with the human exosome subunit PM/Scl100 and are involved in pre-rRNA processing, Nucleic Acids Res 35 (2007) 2564-2572.

[49] A. van Hoof, R.R. Staples, R.E. Baker, R. Parker, Function of the ski4p (Cs14p) and Ski7p proteins in 3'to-5' degradation of mRNA, Mol Cell Biol 20 (2000) 8230-8243.

[50] Y. Araki, S. Takahashi, T. Kobayashi, H. Kajiho, S. Hoshino, T. Katada, Ski7p G protein interacts with the exosome and the Ski complex for 3'-to-5' mRNA decay in yeast, Embo J 20 (2001) 4684-4693.

[51] J.T. Brown, X. Bai, A.W. Johnson, The yeast antiviral proteins Ski2p, Ski3p, and Ski8p exist as a complex in vivo, Rna 6 (2000) 449-457.

[52] S.A. Synowsky, A.J. Heck, The yeast Ski complex is a hetero-tetramer, Protein Sci 17 (2008) 119-125.

[53] S. Takahashi, Y. Araki, T. Sakuno, T. Katada, Interaction between Ski7p and Upf1p is required for nonsense-mediated 3'-to-5' mRNA decay in yeast, Embo J 22 (2003) 3951-3959.

[54] H.H. Hau, R.J. Walsh, R.L. Ogilvie, D.A. Williams, C.S. Reilly, P.R. Bohjanen, Tristetraprolin recruits functional mRNA decay complexes to ARE sequences, J Cell Biochem 100 (2007) 1477-1492.

[55] R. Gherzi, K.Y. Lee, P. Briata, D. Wegmuller, C. Moroni, M. Karin, C.Y. Chen, A KH domain RNA binding protein, KSRP, promotes ARE-directed mRNA turnover by recruiting the degradation machinery, Mol Cell 14 (2004) 571-583.

[56] J. Lykke-Andersen, E. Wagner, Recruitment and activation of mRNA decay enzymes by two AREmediated decay activation domains in the proteins TTP and BRF-1, Genes Dev 19 (2005) 351-361.

[57] G. Stoecklin, T. Mayo, P. Anderson, ARE-mRNA degradation requires the 5'-3' decay pathway, EMBO Rep 7 (2006) 72-77.

[58] S. Phillips, J.S. Butler, Contribution of domain structure to the RNA 3' end processing and degradation functions of the nuclear exosome subunit Rrp6p, Rna 9 (2003) 1098-1107.

[59] X. Wang, H. Jia, E. Jankowsky, J.T. Anderson, Degradation of hypomodified tRNAi Met in vivo involves RNA-dependent ATPase activity of the DExH helicase Mtr4p, Rna 14 (2008) 107-116.

[60] E. Jankowsky, C.H. Gross, S. Shuman, A.M. Pyle, Active disruption of an RNA-protein interaction by a DExH/D RNA helicase, Science 291 (2001) 121-125.

[61] M.E. Fairman, P.A. Maroney, W. Wang, H.A. Bowers, P. Gollnick, T.W. Nilsen, E. Jankowsky, Protein displacement by DExH/D "RNA helicases" without duplex unwinding, Science 304 (2004) 730-734.

[62] M. Malecki, R. Jedrzejczak, P.P. Stepien, P. Golik, In vitro Reconstitution and Characterization of the Yeast Mitochondrial Degradosome Complex Unravels Tight Functional Interdependence, J Mol Biol 372 (2007) 23-36.

[63] E.J. Steinmetz, N.K. Conrad, D.A. Brow, J.L. Corden, RNA-binding protein Nrd1 directs poly(A)independent 3'-endformation of RNA polymerase II transcripts, Nature 413 (2001) 327-331.

[64] M. Kim, L. Vasiljeva, O.J. Rando, A. Zhelkovsky, C. Moore, S. Buratowski, Distinct Pathways for snoRNA and mRNA Termination, Mol Cell 24 (2006) 723-734.

[65] K.L. Carroll, R. Ghirlando, J.M. Ames, J.L. Corden, Interaction of yeast RNA-binding proteins Nrd1 and Nab3 with RNA polymerase II terminator elements, Rna 13 (2007) 361-373.

[66] J.T. Arigo, D.E. Eyler, K.L. Carroll, J.L. Corden, Termination of cryptic unstable transcripts is directed by yeast RNA-binding proteins Nrd1 and Nab3, Mol Cell 23 (2006) 841-851.

[67] M. Thiebaut, E. Kisseleva-Romanova, M. Rougemaille, J. Boulay, D. Libri, Transcription termination and nuclear degradation of cryptic unstable transcripts: a role for the nrd1-nab3 pathway in genome surveillance, Mol Cell 23 (2006) 853-864.

[68] L. Vasiljeva, S. Buratowski, Nrd1 interacts with the nuclear exosome for 3' processing of RNA polymerase II transcripts, Mol Cell 21 (2006) 239-248.

[69] Z. Li, S. Reimers, S. Pandit, M.P. Deutscher, RNA quality control: degradation of defective transfer RNA, Embo J 21 (2002) 1132-1138.

[70] S. Kadaba, X. Wang, J.T. Anderson, Nuclear RNA surveillance in Saccharomyces cerevisiae: Trf4pdependent polyadenylation of nascent hypomethylated tRNA and an aberrant form of 5S rRNA, Rna 12 (2006) 508-521. 
[71] J.A. Stead, J.L. Costello, M.J. Livingstone, P. Mitchell, The PMC2NT domain of the catalytic exosome subunit Rrp6p provides the interface for binding with its cofactor Rrp47p, a nucleic acid-binding protein, Nucleic Acids Res (2007).

[72] P. Mitchell, E. Petfalski, R. Houalla, A. Podtelejnikov, M. Mann, D. Tollervey, Rrp47p is an exosomeassociated protein required for the 3' processing of stable RNAs, Mol Cell Biol 23 (2003) 6982-6992.

[73] N. Suzuki, E. Noguchi, N. Nakashima, M. Oki, T. Ohba, A. Tartakoff, M. Ohishi, T. Nishimoto, The Saccharomyces cerevisiae small GTPase, Gsp1p/Ran, is involved in 3' processing of 7S-to-5.8S rRNA and in degradation of the excised 5'-A0 fragment of 35S pre-rRNA, both of which are carried out by the exosome, Genetics 158 (2001) 613-625.

[74] J.T. Arigo, K.L. Carroll, J.M. Ames, J.L. Corden, Regulation of yeast NRD1 expression by premature transcription termination, Mol Cell 21 (2006) 641-651.

[75] K.A. Kopcewicz, W. O'Rourke T, D. Reines, Metabolic Regulation of IMD2 Transcription and an Unusual DNA Element That Generates Short Transcripts, Mol Cell Biol 27 (2007) 2821-2829.

[76] C.C. Reis, J.L. Campbell, Contribution of Trf4/5 and the nuclear exosome to genome stability through regulation of histone mRNA levels in Saccharomyces cerevisiae, Genetics 175 (2007) 993-1010.

[77] F. Ibrahim, J. Rohr, W.J. Jeong, J. Hesson, H. Cerutti, Untemplated oligoadenylation promotes degradation of RISC-cleaved transcripts, Science 314 (2006) 1893. 


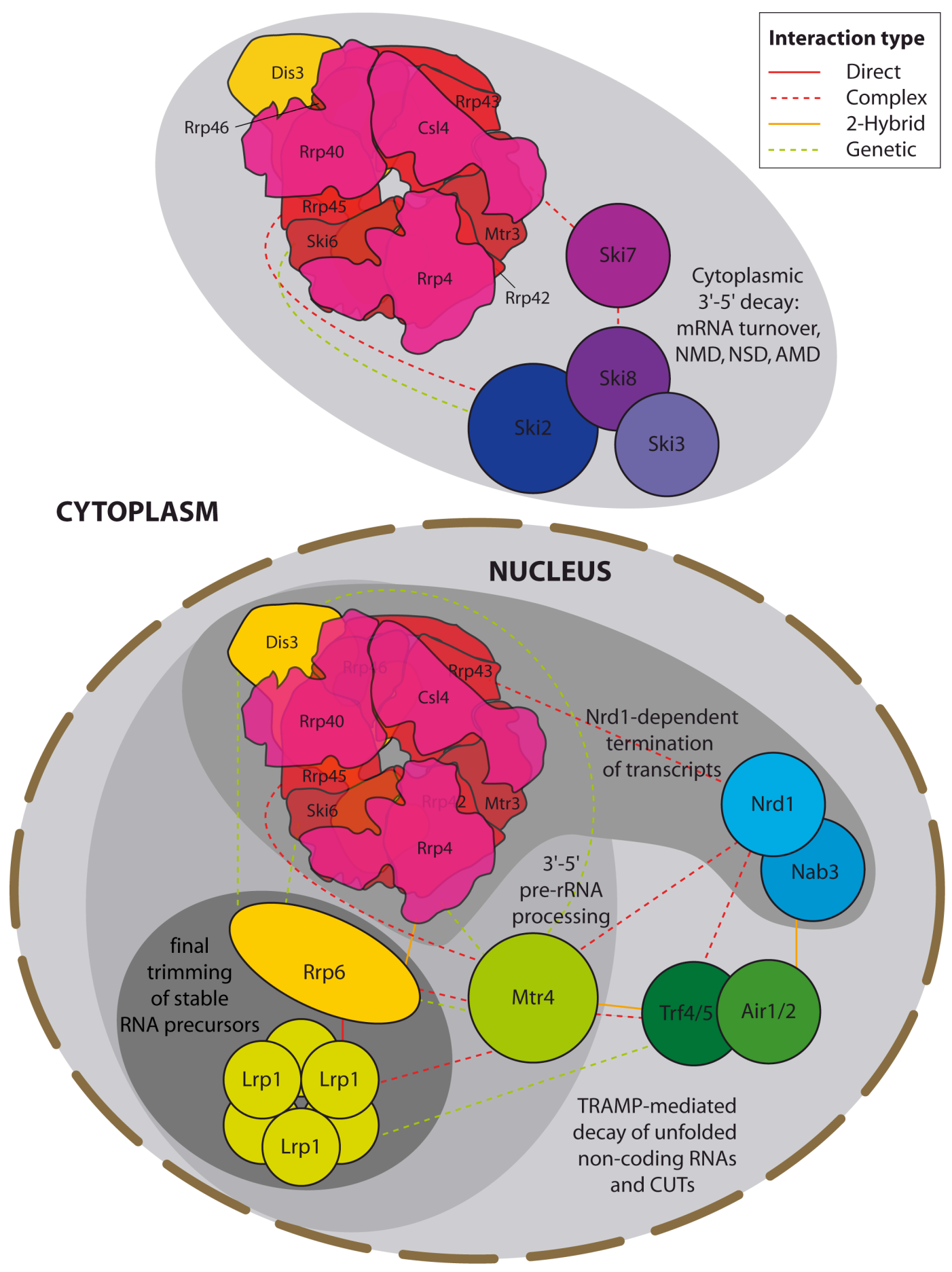

Figure 1. Interaction network between the yeast nuclear and cytoplasmic exosomes and their cofactors. Each reported interaction between several factors is represented by a plain or dashed colour line. "Direct" interaction designate in vitro-reconstituted complexes. "Complex" refers to various techniques of protein complex purification by affinity chromatography. "Genetic" interactions are considered in bulk, regardless of their nature (i.e. synthetic lethal, dosage rescue, etc.). For more convenience, factors that are known to form complexes with a defined stoichiometry are grouped together, and lines are eluded; when affinity-purification data link one factor with several members of a same complex, only one interaction is represented. All factors taking part in a common biological process are gathered within the same grey unit. 